\title{
The Long Noncoding RNA NEAT1 Targets miR-34a-5p and Drives Nasopharyngeal Carcinoma Progression via Wnt/ $\beta$-Catenin Signaling
}

\author{
Yuqing Ji ${ }^{1}$, Man Wang ${ }^{1}$, Xueshen $\mathrm{Li}^{1}$, and Fusheng Cui ${ }^{2}$ \\ ${ }^{1}$ Ear-Nose-Throat Department, Xingtai People's Hospital, Xingtai; \\ ${ }^{2}$ CT/MRI Department, Xingtai People's Hospital, Xingtai, China.
}

\begin{abstract}
Purpose: Long noncoding RNA nuclear paraspeckle assembly transcript 1 (NEAT1) has been deemed an oncogene in many human cancers. However, the underlying mechanism of NEAT1 in nasopharyngeal carcinoma (NPC) progression remains largely unclear.

Materials and Methods: Quantitative real-time PCR assay was performed to assess the expression of NEAT1 and miR-34a-5p in NPC tissues and cells. Western blot analysis was used to observe cell epithelial to mesenchymal transition (EMT) and the activation of Wnt/ $\beta$-catenin signaling in 5-8F cells. MiRNA directly interacting with NEAT1 were verified by dual-luciferase reporter assay and RNA immunoprecipitation. Cell proliferation ability was determined by CCK-8 assay, and cell migration and invasion capacities were assessed by transwell assays. An animal model was used to investigate the regulatory effect of NEAT1 on tumor growth in vivo.

Results: Our data revealed that NEAT1 is upregulated, while miR-34a-5p is downregulated in NPC tissues and cell lines. NEAT1 knockdown repressed tumor growth in vitro and in vivo. Additionally, we discovered that NEAT1 directly binds to miR-34a-5p and suppresses miR-34a-5p expression. Moreover, NEAT1 knockdown exerted suppression effects on cell proliferation, migration, invasion, and EMT by miR-34a-5p. NEAT1 knockdown blocked Wnt/ $\beta$-catenin signaling via miR-34a-5p.

Conclusion: Our study demonstrated that NEAT1 targets miR-34a-5p at least partly to drive NPC progression by regulating Wnt/ $\beta$-catenin signaling, suggesting a potential therapeutic target for NPC.
\end{abstract}

Key Words: Nasopharyngeal carcinoma, lncRNA NEAT1, miR-34a-5p, Wnt/ $\beta$-catenin signaling pathway

\section{INTRODUCTION}

Nasopharyngeal carcinoma (NPC), a common head and neck cancer arising from the nasopharynx epithelium, is a leading cause of cancer-related death worldwide. ${ }^{1}$ NPC is prevalent in the east and southeast parts of Asia and the east and northern

Received: October 26, 2018 Revised: January 5, 2019

Accepted: January 15, 2019

Corresponding author: Fusheng Cui, MM, CT/MRI Department, Xingtai People's Hospital, No.16, Hongxing Street, Oiaodong District, Xingtai, Hebei, 054001, China. Tel: 86-0319-3286450, E-mail: yuanlisoly@163.com

-The authors have no potential conflicts of interest to disclose.

(C) Copyright: Yonsei University College of Medicine 2019

This is an Open Access article distributed under the terms of the Creative Commons Attribution Non-Commercial License (https://creativecommons.org/licenses/ by-nc/4.0) which permits unrestricted non-commercial use, distribution, and reproduction in any medium, provided the original work is properly cited.
Africa. ${ }^{1}$ Many risk factors are known to contribute to the pathogenesis of NPC, such as Epstein-Barr virus infection, genetic susceptibility, and tobacco addiction. ${ }^{2}$ Irradiation therapy, alone or combined with chemotherapy, is a widely used modality of NPC treatment. ${ }^{3}$ Although development of therapeutic strategies has improved treatment effects, the prognosis of NPC patients still remains poor. ${ }^{4}$ Hence, it is important to search for novel prognostic biomarkers and therapeutic targets for NPC treatment.

Long noncoding RNAs (IncRNAs), defined as transcripts longer than $200 \mathrm{nt}$ with no protein-coding potential, are recognized as playing an important role in the fundamental processes involved in life. ${ }^{5}$ By interacting with cellular macromolecules, such as DNA, RNA, and protein, IncRNAs can drive a series of vital tumor phenotypes. ${ }^{6}$ Accumulating evidence suggests that some cancer-associated IncRNAs hold the po- 
tential for further applications in the fight against human cancer. ${ }^{7,8}$ Nuclear paraspeckle assembly transcript 1 (NEAT1), transcribed from the familial tumor syndrome multiple endocrine neoplasia type 1 located on chromosome 11, is an essential structural component of paraspeckles. ${ }^{9}$ Recently, NEAT1 has been determined as an oncogene in many human cancers, such as laryngeal squamous cell cancer, ${ }^{10}$ gastric cancer, ${ }^{11}$ and ovarian cancer. ${ }^{12}$ Additionally, NEAT1 was reported to be upregulated in NPC and to enhance the tumorigenesis and progression of NPC by modulating miR-124/NF- $\kappa$ B signaling. ${ }^{13}$ Moreover, NEAT1 overexpression was shown to be tightly linked to epithelial to mesenchymal transition (EMT) and radioresistance of NPC cells by regulating the miR-204/ ZEB1 axis. ${ }^{14}$ However, the underlying mechanism of NEAT1 on NPC progression has still remained largely unclear.

MicroRNAs (miRNAs), a conserved class of small non-coding RNAs of approximately $22 \mathrm{nt}$ in length, can post-transcriptionally regulate gene expression, thereby modulating downstream signaling pathways and affecting tumorigenesis and cancer progression. ${ }^{15}$ Growing evidence has suggested that a number of miRNAs play crucial roles in the pathogenesis of NPC: For instance, miR-185 repressed cell proliferation and enhanced apoptosis and autophagy by regulating HOXC6 and TGF- $\beta 1 /$ mTOR in NPC. ${ }^{16}$ MiR-342 targeted ZEB1 to suppress the proliferation and invasion in NPC cells. ${ }^{17}$ Moreover, miR$10 \mathrm{~b}$ promoted the acquisition of an EMT phenotype by regulating KLF4/Notch1/E-cadherin in cisplatin-resistant NPC cells. ${ }^{18}$ In the present study, we validated whether NEAT1 levels are elevated in NPC tissues and cells. In doing so, we determine that NEAT1 targets miR-34a-5p to drive NPC progression, at least in part, by regulating Wnt/ $\beta$-catenin signaling pathway.

\section{MATERIALS AND METHODS}

\section{Tissues collection}

A total of 33 pairs of NPC tissues and adjacent normal tissues were obtained from NPC patients receiving radical surgery at Xingtai People's Hospital, Hebei Province. Among these patients, 15 were male and 18 were female. Lymph node metastasis was encountered in 19 cases, but not in 14 cases. Twenty cases were stage I or II, and 13 cases were stage III or IV in terms of TNM staging. In addition, the average age was $46.14 \pm 0.58$ years. All tissues were collected and stored at $-80^{\circ} \mathrm{C}$ before experimentation. For survival assay, we divided the 33 patients into two groups: low NEAT1 expression group ( $n=14$, $\geq$ medium value) and high NEAT1 expression group $(n=19$, $<$ medium value) according to the medium value of NEAT1 expression in NPC tissues. Prior written informed consent was provided by each patient, and the study was approved by the Ethics Committee of Xingtai People's Hospital, Hebei Province.

\section{Cell culture}

Five NPC cell lines (CNE1, CNE2, SUNE1, SUNE2, and 5-8F) and the human nasopharyngeal epithelial cell line NP69 were purchased from the American Type Culture Collection (ATCC, Manassas, VA, USA). All cells were grown in RPMI-1640 medium (Gibco, Rockville, MD, USA) containing 10\% FBS (Gemini Bio-Products, West Sacramento, CA, USA) and 1\% penicillin/ streptomycin (Gibco) in a humidified $37^{\circ} \mathrm{C}$ incubator supplemented with $5 \% \mathrm{CO}_{2}$.

\section{Cell transfection}

MiRNA mimics for miR-34a-5p (miR-34a-5p mimics) and negative control miR-NC, miR-34a-5p inhibitor (in-miR-34a$5 p)$, negative control in-miR-NC, NEAT1-special small interfering RNA (si-NEAT1), and negative control si-NC were obtained from GenePhama (Shanghai, China). The NEAT1 overexpression vector (pcDNA-NEAT1) was commercially constructed by Sigma-Aldrich (St. Louis, MO, USA), and empty pcDNA vector was used as the negative control. When cells reached $60-80 \%$ confluence, transient transfection was performed using Lipofectamine 2000 transfection reagent (Life Technologies, Carlsbad, CA, USA) referring to manufacturer's instructions.

\section{RNA isolation and quantitative real-time PCR}

Total RNA was freshly extracted from tissues and cells using a miRNeasy FFPE kit (Qiagen, Valencia, CA, USA). Quality of the isolated RNA was assessed by a NanoDrop 2000 spectrophotometer (Thermo Fisher Scientific, Waltham, MA, USA). For NEAT1 detection, RNA samples were reversely transcribed into cDNA with Superscript III Reverse Transcriptase (Invitrogen, Waltham, MA, USA). Then, quantitative real-time PCR (qRT-PCR) assay was performed using a SuperScript Platinum SYBR ${ }^{\mathrm{TM}}$ Green One-Step qRT-PCR Kit (Invitrogen) on a 7900HT Fast Real-Time PCR System (Thermo Fisher Scientific), with normalization to the expression of GAPDH. Fold changes were calculated using the $2^{-\Delta \Delta \mathrm{Ct}}$ method. For miR34a-5p detection, cDNA was synthesized using miScript II RT Kit (Qiagen), and qRT-PCR was performed by miScript SYBR Green PCR Kit (Qiagen) and miScript Primer Assay specific for miR-34a-5p (Qiagen). U6 was used as an endogenous control.

\section{Dual-luciferase reporter assay}

Online software Lncbase v.2 (http://carolina.imis.athena-innovation.gr/diana_tools/web/index.php?r=site\%2Ftools) was used to predict miRNAs directly interacting with NEAT1. Fragments of the NEAT1 sequence containing potential miR-34a$5 \mathrm{p}$ complementary sites or those with mutational miR-34a-5p target sites were inserted into psiCHECK-2 vector (Promega, Madison, WI, USA) to construct NEAT1 wild-type (WT) or mutant-type (MUT) reporter vector. The constructs were transiently cotransfected into $5-8 \mathrm{~F}$ cells with miR-34a-5p mimics or in-miR-34a-5p. After $48 \mathrm{~h}$ transfection, the signals of firefly 
and Renilla luciferase in cell lysates were measured using a dual-luciferase reporter assay system (Promega), and relative luciferase activities were calculated as the ratio of Renilla/firefly luciferase activity.

\section{RNA immunoprecipitation assay}

RIP assay was performed to explore whether RNA induced silencing complex (RISC) contained NEAT1 and miR-34a-5p in 5-8F cells using an Imprint RNA Immunoprecipitation (RIP) kit (Millipore, Billerica, MA, USA) referring to the directions of the manufacturers. In brief, 5-8F cells transfected with miR34a-5p mimics were lysed in RIP lysis buffer (Takara, Beijing, China) and were incubated with protein $\mathrm{A} / \mathrm{G}$ magnetic beads and Argonaute-2 antibody (anti-Ago2, Abcam, Cambridge, UK) or IgG antibody (anti-IgG, Abcam). Then, cell lysates in the miR-34a-5p mimics group were centrifuged. The supernatants were marked as output, and immunoprecipitation complexes were named as IP. Western blot analysis was performed to validate successful RIP with anti-Ago2. Lastly, qRT-PCR assay was used to detect NEAT1 enrichment in the RIP RNA fraction.

\section{Cell proliferation assay}

Cell proliferation capacity was measured with the Cell Counting kit-8 (CCK-8, Dojindo Laboratories, Kumamoto, Japan) referring to the manufacturer's protocol. Briefly, cells were seeded in 96-well plates and transfected with si-NEAT1 or siNEAT1+in-miR-34a-5p. After 0, 24, 48, 72, and 96 hours transfection, $10 \mu \mathrm{L}$ of CCK-8 solution was added into each well, followed by determination of absorbance at $450 \mathrm{~nm}$ using a MRX II microplate reader (Dynex Technologies, Chantilly, VA, USA).

\section{Cell migration assay}

Cell migration assay was carried out using a 24-Transwell plate with $8-\mu \mathrm{m}$ pores (Corning Incorporated-Life Sciences, Tewksbury, MA, USA). To be brief, 5-8F cells were transfected with si-NEAT1 alone or together with in-miR-34a-5p for $48 \mathrm{~h}$. Then, transfected cells $\left(1.0 \times 10^{5}\right)$ were seeded in the upper chamber, and $500 \mu \mathrm{L}$ of growth medium containing 10\% FBS was added in the lower chamber. After incubation for $48 \mathrm{~h}$ at $37^{\circ} \mathrm{C}$, traversed cells on the lower side were fixed in $4 \%$ phosphate-buffered formaldehyde (PFA) and stained with $0.1 \%$ crystal violet (Sigma-Aldrich, St. Louis, MO, USA). At last, images were obtained, and migrated cells were counted using a microscope (Leica, Wetzlar, Germany) with Leica Qwin IM500 software.

\section{Cell invasion assay}

Cell invasion ability was assessed using Transwell filters precoated with $50 \mathrm{ng} / \mathrm{mL}$ of Matrigel (BD Bioscience, San Jose, CA, USA). Transfected cells $\left(1.0 \times 10^{5}\right)$ were seeded into the upper chamber, and the bottom chamber was filled with 500 $\mu \mathrm{L}$ of growth medium containing $10 \%$ FBS. After incubation for $48 \mathrm{~h}$ at $37^{\circ} \mathrm{C}$, invaded cells were fixed in $4 \%$ PFA and stained with $0.1 \%$ crystal violet.

\section{Western blot}

Total protein was extracted in chilled lysis buffer comprising $50 \mathrm{mM}$ Tris- $\mathrm{HCl}$ (pH=7.4), 1 mM EDTA, 1\% Triton X-100, 150 $\mathrm{mM} \mathrm{NaCl}, 0.1 \%$ SDS, $0.1 \%$ deoxycholic acid, and $1 \%$ Protease Inhibitor Cocktail (Sigma-Aldrich). Protein extract concentrations were measured using BCA Protein Assay Kits (Pierce, Rockford, IL, USA). Ten micrograms of protein extract were separated by $10 \%$ SDS/polyacrylamide gel electrophoresis and then electroblotted onto a PVDF membranae (Du Pont, Boston, MA, USA). After blockage with 5\% nonfat milk in TBS containing $0.1 \%$ Tween 20 (TBST), membranes were hybridized with antibodies against E-cadherin (1:1000, Cell Signaling Technology, Danvers, MA, USA), N-cadherin (1:1000, Cell Signaling Technology), Vimentin (1:1000, Cell Signaling Technology), $\beta$-catenin (1:5000, Abcam), cyclin D1 (1:10000, Abcam), c-myc (1:1000, Abcam), Ago2 (1:1000, Abcam), and $\beta$-actin (1:1000, Cell Signaling Technology) overnight at $4^{\circ} \mathrm{C}$. After washing three times with TBST, the membranes were further incubated by horseradish-peroxidase-linked immunoglobulin G (Cell Signaling Technology) and visualized by a chemiluminescence detection kit (New England Biolabs, Beverly, MA, USA) on the Enhanced Chemiluminescence Western blot system (GE Healthcare Bioscience, Tokyo, Japan) with ImageJ software (National Institutes of Health, Bethesda, MD, USA).

\section{sh-NEAT1 cell construct}

Lentiviruses with or without a NEAT1 knockout sequence (shNEAT1 or sh-NC) were constructed by Sigma-Aldrich, and were used to infect 5-8F cells. Puromycin was used to screened the stable sh-NEAT1-infected cells.

\section{In vivo animal model}

Female 6-week old SCID mice were purchased from Beijing Vital River Laboratory Animal Technology Corporation (Beijing, China) and housed in a specific-pathogen-free environment. Approximately $1.0 \times 10^{7} \mathrm{sh}-\mathrm{NEAT} 1 / \mathrm{sh}-\mathrm{NC}$-infected cells were subcutaneously inoculated into the nude mice to form xenograft mice $(n=10)$. Tumor volume was measured with a caliper every one week. At 5 weeks after injection, all mice were euthanized and tumor tissues were excised for weight evaluation, qRT-PCR, and western blot assays. All experimental processes were approved by the Animal Care and Use Committee of Xingtai People's Hospital, Hebei Province.

\section{Statistical analysis}

All analyses were carried out using SPSS 18.0 software (SPSS Inc., Chicago, IL, USA). Paired Student's t-test, Mann-Whitney $\mathrm{U}$ test, and one-way analysis of variance were used in the study to evaluate statistical differences. $p<0.05$ was considered 
statistically significant.

\section{RESULTS}

\section{Upregulation of NEAT1 in NPC tissue and cell lines}

To validate the function of NEAT1 in NPC progression, NEAT1 expression was first measured by qRT-PCR assay in NPC tissues and adjacent non-cancerous nasopharynx tissues. The data revealed that NEAT1 expression is highly increased in NPC tissues, compared with corresponding controls (Fig. 1A and B). Subsequently, we assessed the expression of NEAT1 in NPC cell lines. Consistently, NEAT1 levels were significantly higher in NPC cell lines than those in NP69 cells (Fig. 1C).

We then sought to determine whether NEAT1 expression is associated with the prognosis of NPC patients. According to the medium value of NEAT1 expression, 33 patients were divided into two groups: low NEAT1 expression group $(n=14)$ and high NEAT1 expression group ( $\mathrm{n}=19)$. Kaplan-Meier survival analysis revealed that low NEAT1 expression afforded longer overall survival time that high NEAT1 expression (Fig. 1D) (log-rank $p<0.05$ ). All of these results supported that NEAT1 played an oncogenic role in the progression of NPC.

\section{Correlations between NEAT1 expression and clinical characteristics}

Then, we further explored correlations between NEAT1 and clinical characteristics. As shown in Table 1, NEAT1 expres-

Table 1. Analysis of the Correlations between NEAT1 Expression in Primary Nasopharyngeal Carcinoma and Clinicopathologic Parameters

\begin{tabular}{|c|c|c|c|c|}
\hline \multirow{2}{*}{ Variable } & \multirow{2}{*}{$\begin{array}{l}\text { Patients } \\
\text { (n) }\end{array}$} & \multicolumn{2}{|c|}{ NEAT1 expression } & \multirow{2}{*}{$p$ value } \\
\hline & & Low & High & \\
\hline Sex & & & & 0.827 \\
\hline Male & 15 & 8 & 7 & \\
\hline Female & 18 & 6 & 12 & \\
\hline Age (yr) & & & & 0.318 \\
\hline$<45$ & 16 & 7 & 9 & \\
\hline$\geq 45$ & 17 & 7 & 10 & \\
\hline Tumor stage & & & & $0.025^{*}$ \\
\hline I, II & 20 & 9 & 11 & \\
\hline III, IV & 13 & 5 & 8 & \\
\hline Lymph node status & & & & $0.004^{\dagger}$ \\
\hline Metastasis & 19 & 8 & 11 & \\
\hline No metastasis & 14 & 6 & 8 & \\
\hline
\end{tabular}

NEAT1, nuclear paraspeckle assembly transcript 1.

${ }^{*} p<0.05$ and ${ }^{\dagger} p<0.01$ were considered statistically significant.
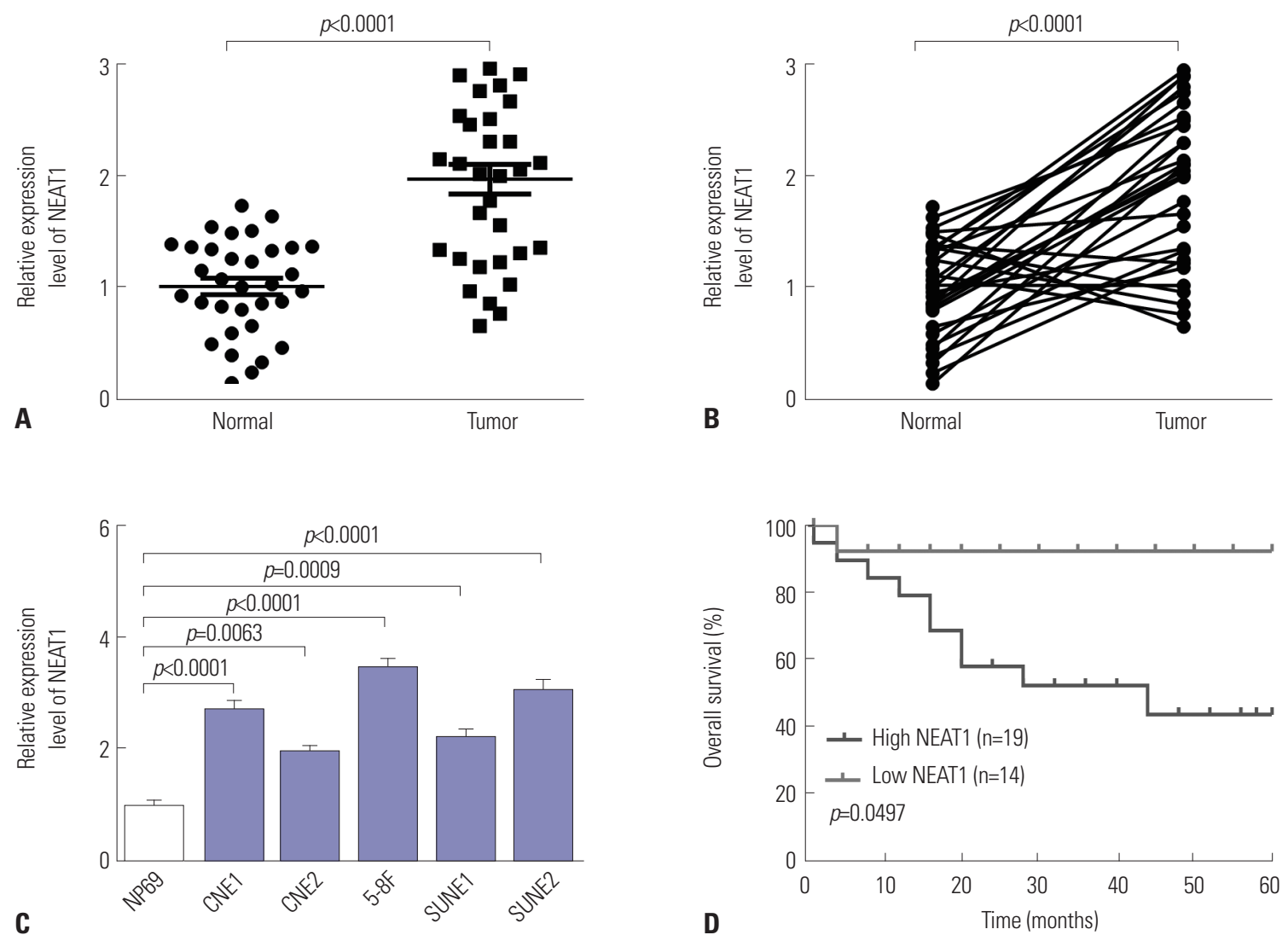

Fig. 1. Upregulation of NEAT1 expression in NPC tissues and cell lines. (A and B) NEAT1 expression was detected by qRT-PCR assay in NPC tissues and corresponding normal tissues. (C) qRT-PCR assay of NEAT1 expression in nasopharyngeal epithelial cells (NP69) and NPC cell lines (CNE1, CNE2, SUNE1, $5-8 \mathrm{~F}$ and SUNE2). (D) Kaplan-Meier survival method and log-rank test were performed to analyze the association between NEAT1 level and prognosis of NPC patients. NEAT1, nuclear paraspeckle assembly transcript 1; NPC, nasopharyngeal carcinoma. 

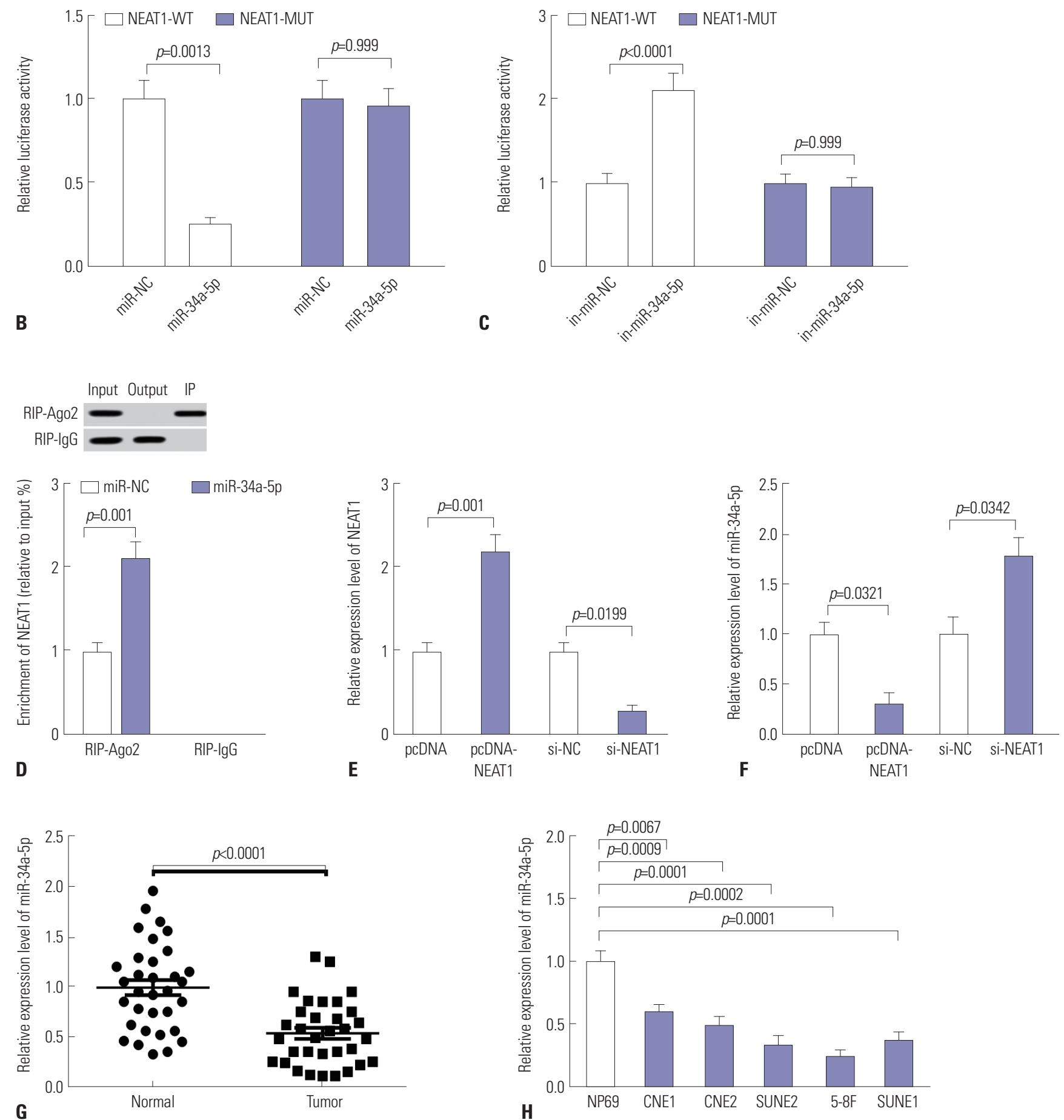

Fig. 2. NEAT1 directly binds to miR-34a-5p and suppresses miR-34a-5p expression. (A) The predicted miR-34a-5p binding sequence within NEAT1 wildtype (WT) and a mutated form (MUT). 5-8F cells were cotransfected with NEAT1-WT or NEAT1-MUT and miR-NC mimics, miR-34a-5p mimics (B), in-miR$\mathrm{NC}$, and in-miR-34a-5p (C), followed by measurement of relative luciferase activity. (D) RIP experiment was performed using anti-Ago2 in 5-8F cells transfected with miR-34a-5p mimics and anti-IgG as a negative control, and Western blot analysis was used to validate successful RIP on the Enhanced Chemiluminescence Western blot system with ImageJ software. 5-8F cells were transfected with pcDNA, pcDNA-NEAT1, si-NC, or si-NEAT1, followed by detection of NEAT1 (E) and miR-34a-5p (F) expression levels. MiR-34a-5p expression was assessed in NPC tissues (G) and cell lines (H). NEAT1, nuclear paraspeckle assembly transcript 1; NPC, nasopharyngeal carcinoma; IP, immunoprecipitation complexes. 
sion was highly associated with TNM staging and lymph node metastasis. No other clinical characteristics were found to be significantly associated with NEAT1 expression.

\section{NEAT1 directly binds to miR-34a-5p and suppresses miR-34a-5p expression}

To further explore the underlying mechanism of NEAT1 as an oncogene in NPC progression, Lncbase v. 2 was explored to search for target miRNAs binding to NEAT1. The predicted data indicated that NEAT1 harbors a putative binding region with miR-34a-5p (Fig. 2A). Thus, dual-luciferase reporter assay and RIP assay were employed to verify whether NEAT1 directly binds to miR-34a-5p in NPC cells. NEAT1-WT or NEAT1-MUT constructs were transfected into 5-8F cells with miR-34a-5p mimics or in-miR-34a-5p, followed by detection of relative luciferase activity. In $5-8 \mathrm{~F}$ cells, the relative luciferase activity of NEAT1-WT was significantly decreased by co- transfection with miR-34a-5p mimics, while it was highly increased when introduced with in-miR-34a-5p (Fig. 2B and C). However, the relative luciferase activity of NEAT1-MUT was not affected in response to alteration of miR-34a-5p levels (Fig. 2B and C). Ago2, a component of the RISC, plays a pivotal role in the mature process of miRNAs. Hence, RIP experiments were performed using anti-Ago2 in 5-8F cells transfected with miR-34a-5p mimics to validate the endogenous interaction between NEAT1 and miR-34a-5p. These results revealed that relative enrichment of NEAT1 was higher in miR$34 a-5 p$ transfection group than in the control group (Fig. 2D). Additionally, we discovered that Ago2 primarily exists in IP samples in the RIP-Ago2 group, but in output samples in the RIP-IgG group, indicating that RIP assay was successfully performed.

Then, NEAT1 overexpression vector (pcDNA-NEAT1) or NEAT1-special siRNA (si-NEAT1) was transfected into 5-8F
A

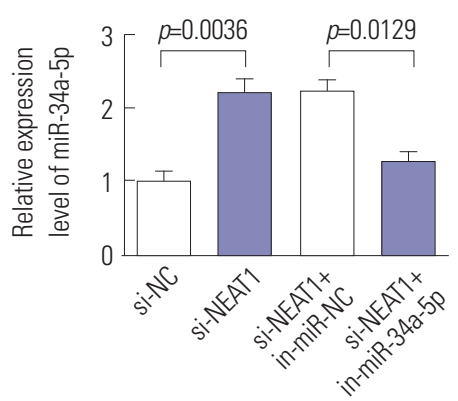

B
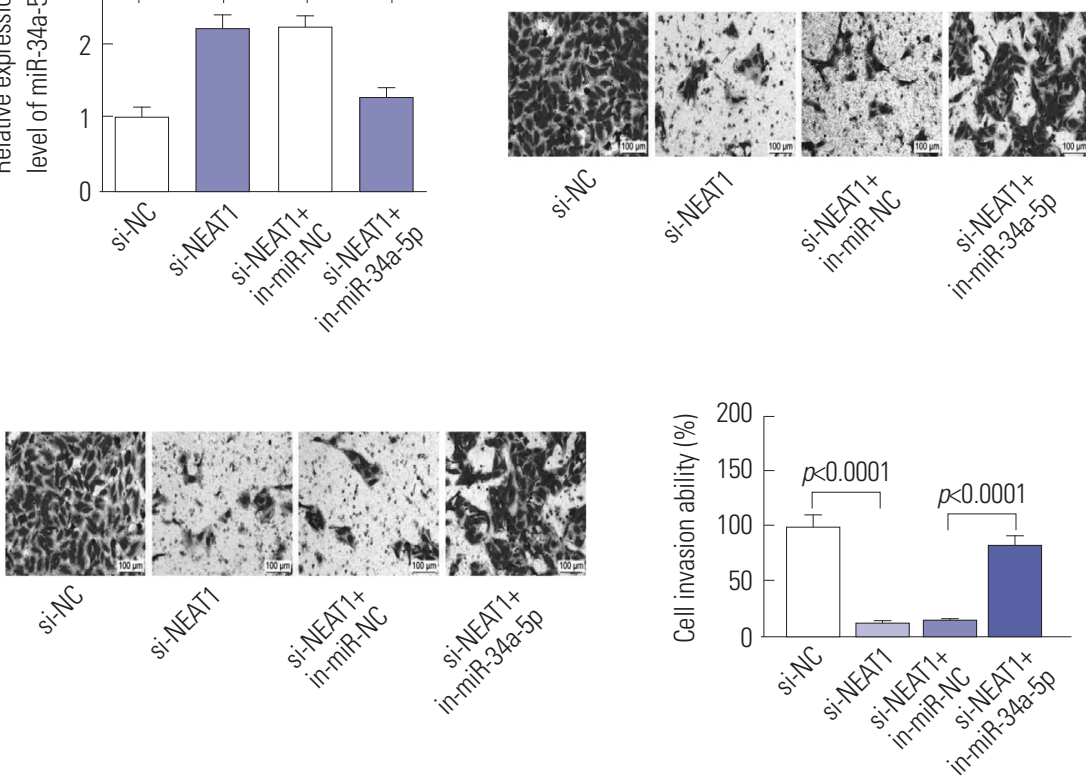

$\mathbf{E}$
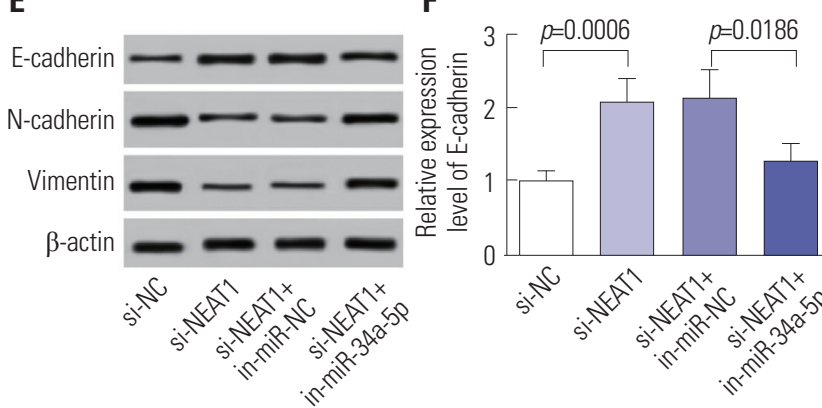

G

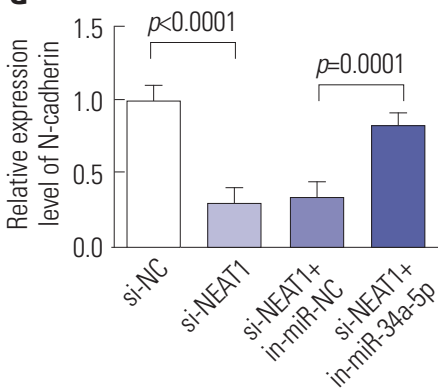

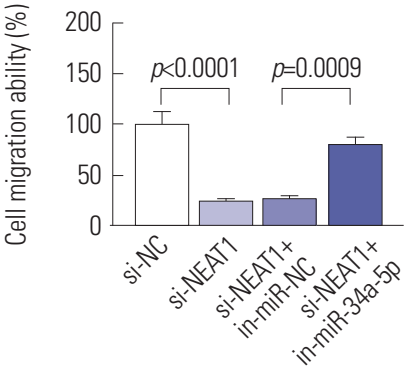

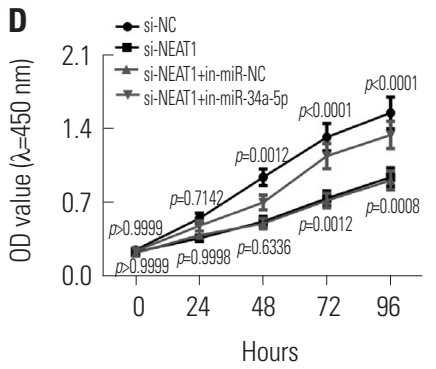

H

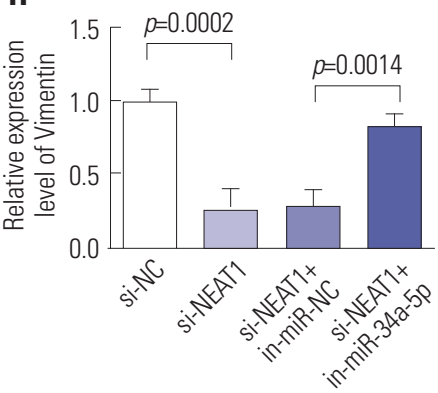

Fig. 3. si-NEAT1 inhibited NPC cell proliferation, migration, invasion, and EMT by miR-34a-5p. 5-8F cells were transfected with si-NC, si-NEAT1, siNEAT1+in-miR-NC, and si-NEAT1+in-miR-34a-5p. (A) qRT-PCR assay of miR-34a-5p expression in treated cells. (B) Transwell assay of cell migration ability in treated cells. (C) Transwell assay of cell invasion capacity in treated cells. (D) CCK-8 assay of cell proliferation ability in treated cells. (E-H) Western blot analysis of E-cadherin, N-cadherin, and Vimentin expression levels in treated cells. Bands were quantified by ImageJ software. NEAT1, nuclear paraspeckle assembly transcript 1; NPC, nasopharyngeal carcinoma; EMT, epithelial to mesenchymal transition. 
cells to observe the effect of NEAT1 on miR-34a-5p expression. As shown in Fig. 2E, NEAT1 levels were markedly elevated by transfection with pcDNA-NEAT1, while they were inhibited by si-NEAT1 transfection. Subsequent experiments revealed that miR-34a-5p expression was significantly and negatively correlated with NEAT1 levels (Fig. 2F). Further, we detected the expression of miR-34a-5p in NPC tissues and cell lines. These data showed that, in comparison to their counterparts, miR-34a-5p levels were strikingly attenuated in NPC tissues and cell lines (Fig. 2G and H). Taken together, these results hinted that NEAT1 might suppress miR-34a-5p expression by directly binding to miR-34a-5p.

\section{si-NEAT1 exerts inhibition effects on cell proliferation,} migration, invasion, and EMT by miR-34a-5p

Next, loss-of-function experiments were performed by transfection of si-NEAT1 into 5-8F cells. Transwell and CCK-8 assays revealed that NEAT1 knockdown remarkably weakened cell migration (Fig. 3B), invasion (Fig. 3C), and proliferation (Fig. 3D), compared with respective controls. In parallel, cell EMT ability was significantly repressed by NEAT1 depletion, presented by an increase of E-cadherin level, as well as de- creases in N-cadherin and Vimentin levels (Fig. 3E-H). These results indicated that NEAT1 knockdown suppresses cell proliferation, migration, invasion, and EMT in NPC in vitro.

We then sought to explore whether the above inhibition effects exerted by NEAT1 knockdown are mediated by miR-34a5p. Hence, by cotransfecting si-NEAT1 and in-miR-34a-5p, functional experiments were conducted in $5-8 \mathrm{~F}$ cells. As shown in Fig. 3A, the si-NEAT1-mediated repression effect on miR-34a-5p expression was strikingly abrogated by cotransfection with in-miR-34a-5p. Subsequent functional experiments demonstrated that si-NEAT1-mediated suppression effects on cell proliferation, migration, invasion, and EMT were significantly reversed by miR-34a-5p expression restoration in 5-8F cells (Fig. 3B-H). These results indicated that NEAT1 knockdown inhibits the proliferation, migration, invasion, and EMT by miR-34a-5p in NPC cell lines.

\section{si-NEAT1 blocks the Wnt/ $\beta$-catenin pathway via miR-34a-5p}

Constitutive activation of $\mathrm{Wnt} / \beta$-catenin signaling pathway has generally been accepted as triggering tumorigenesis and metastasis. ${ }^{19}$ Thus, we further investigated whether NEAT1/
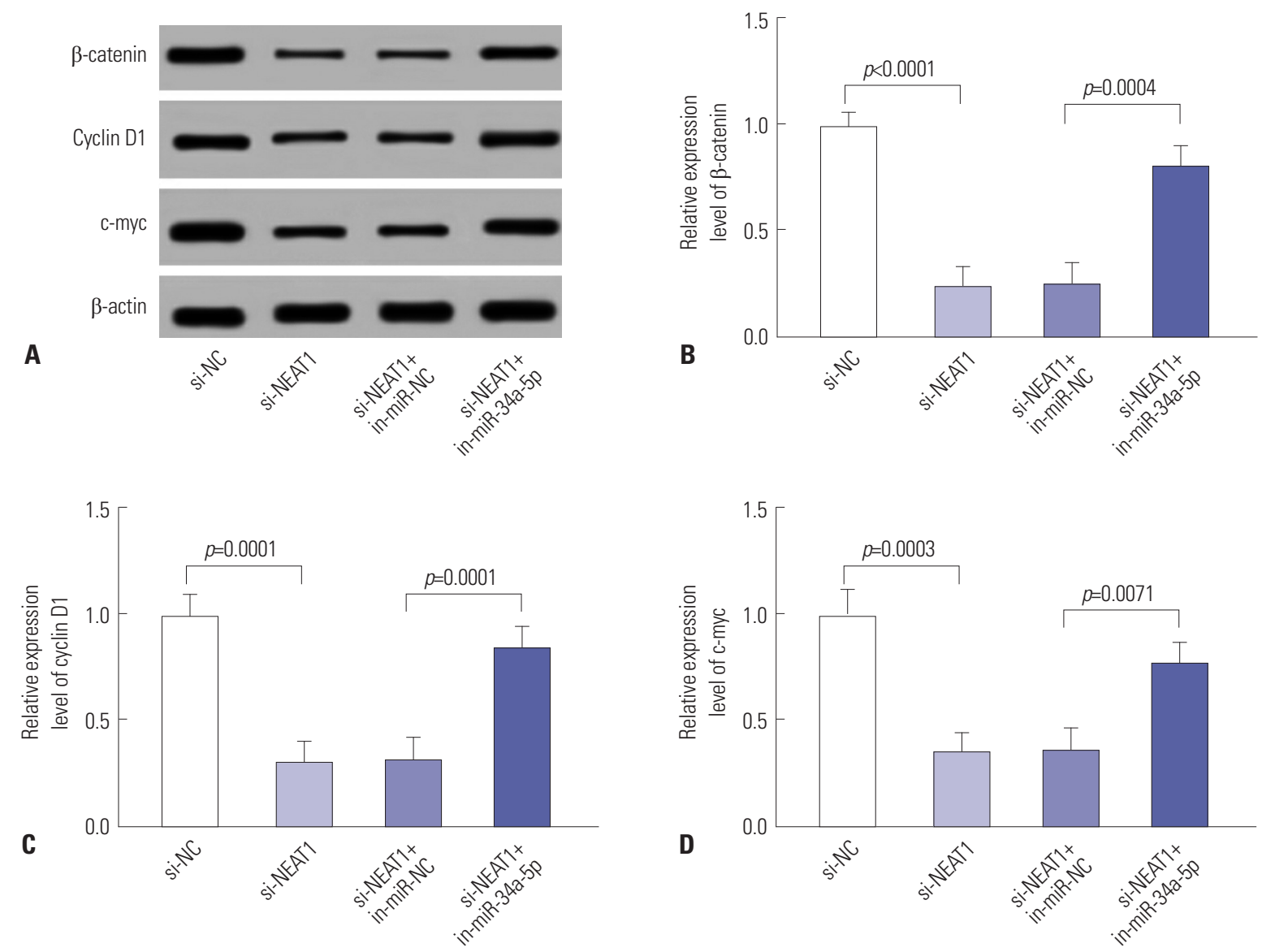

Fig. 4. si-NEAT1 blockaded the Wnt/3-catenin pathway by miR-34a-5p. 5-8F cells were transfected with si-NC, si-NEAT1, si-NEAT1+in-miR-NC, and siNEAT1+in-miR-34a-5p (A), followed by measurement of $\beta$-catenin (B), cyclin D1 (C), and c-myc (D) expression levels by Western blot analysis. Bands were quantified by ImageJ software. NEAT1, nuclear paraspeckle assembly transcript 1. 
miR-34a-5p axis regulates $\mathrm{Wnt} / \beta$-catenin signaling in NPC. 5 -8F cells were transfected with si-NEAT1 alone or together with in-miR-34a-5p, followed by determination of $\beta$-catenin, cyclin D1, and c-myc levels. These data demonstrated that, compared with negative control, NEAT1 knockdown leads to a decrease in $\beta$-catenin, cyclin D1, and c-myc expression levels (Fig. 4), indicating its role as a probable inhibitor of Wnt/ $\beta$-catenin signaling. Furthermore, the inhibitory effect of NEAT1 knockdown was significantly abated by the restoration of miR-34a-5p expression in 5-8F cells. All these results implied that NEAT1 knockdown might blockade Wnt/ $\beta$-catenin signalling by miR-34a-5p in NPC.

\section{NEAT1 knockdown represses tumor growth and EMT by blockading the Wnt/ $\beta$-catenin pathway in vivo}

Lastly, we examined the therapeutic potential of NEAT1 in animal models. These results revealed that sh-NEAT1 infection significantly represses tumor growth, represented by decreases in tumor volume (Fig. 5A) and tumor weight (Fig. 5B). As presented in Fig. 5C and D, sh-NEAT1 introduction markedly weakened NEAT1 expression, but elevated miR-34a-5p levels in xenograft tumors. Moreover, NEAT1 depletion dramatically inhibited EMT in vivo, presented as an increase in E-cadherin level and decreases in $\mathrm{N}$-cadherin and Vimentin levels (Fig. 5E). Additionally, the Wnt/ $\beta$-catenin pathway was inactivated by NEAT1 depletion in tumors derived from shNEAT1-infected cells (Fig. 5F). Together, these findings suggested that NEAT1 knockdown might repress tumor growth and EMT by blockading Wnt/ $\beta$-catenin signaling.

\section{DISCUSSION}

Till now, several lncRNAs, serving as signals of specific cellular states or readouts of active cellular programs, have been shown to be capable of identifying cellular pathologies, such as human cancer. ${ }^{20}$ Moreover, emerging evidence suggests that many lncRNAs are involved in NPC tumorigenesis and progression as an oncogenic gene or a tumor suppressor, providing therapeutic options and prognostic value for NPC patients. For example, MALAT1 was found to be upregulated and to modulate the activity of cancer stem cells and radioresistance by regulating the miR-1/slug axis in NPC. ${ }^{21}$ HOTAIR knockdown was shown to inhibit the expression of fatty acid synthase, thereby repressing cell proliferation and invasion in NPC cells, providing an available therapeutic agent for NPC. ${ }^{22}$ Moreover, LINC01420 overexpression was found to be closely related to an unfavorable prognosis in NPC. ${ }^{23}$ Meanwhile, however, LET was downregulated in NPC tissues, and upregulated LET repressed proliferation of NPC cells and enhanced cell apoptosis. ${ }^{24}$ Ectopic expression of MEG3 resulted in a suppression of tumorigenicity in vivo, indicating its role as a tumor suppressor in NPC. ${ }^{25}$ In this study, our data demonstrated that NEAT1 is upregulated in NPC and that NEAT1 depletion suppresses tumor growth in vivo and in vitro, highlighting its role as an oncogenic gene in NPC.
A

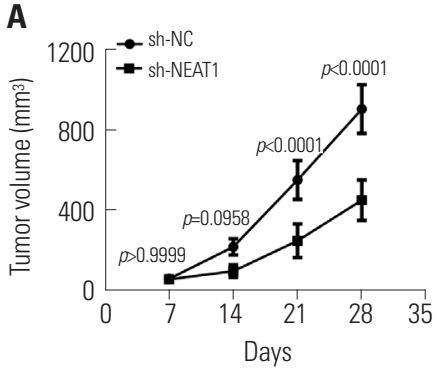

$\mathbf{B}$

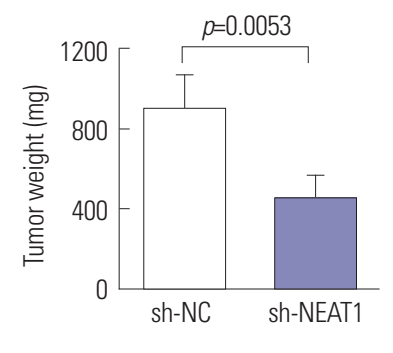

E

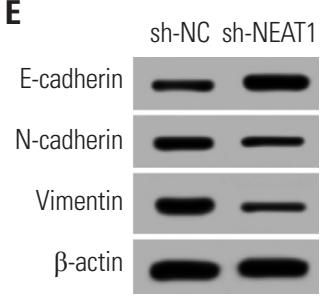

C

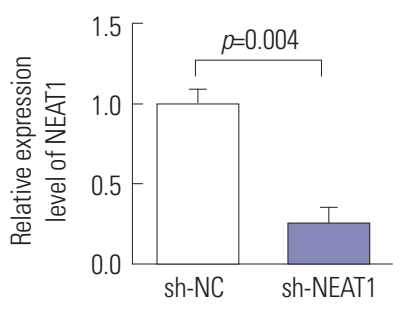

$\mathbf{F}$

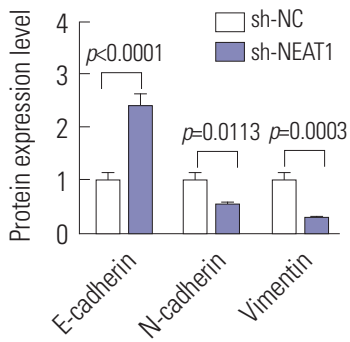

D
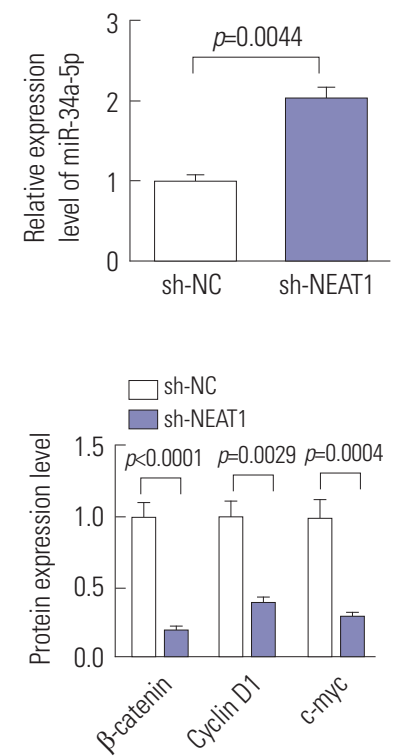

Fig. 5. NEAT1 knockdown represses tumor growth and EMT by Wnt/ $\beta$-catenin signaling in vivo. Nude mice were subcutaneously injected with $1.0 \times 10^{7}$ $5-8 \mathrm{~F}$ cells stably infected by sh-NC or sh-NEAT1, and the mice were euthanized at 5 weeks after implantation. (A) At 7 days after cell implantation, tumor volumes were measured every one week. (B) The average weight of tumors isolated from xenograft mice. qRT-PCR assay of NEAT1 (C) and miR-34a-5p (D) expression levels in excised tumors. Western blot analysis of E-cadherin, N-cadherin, and Vimentin protein levels (E), as well as $\beta$-catenin, cyclin D1, and c-myc expression levels (F), in excised tumor tissues with ImageJ software. NEAT1, nuclear paraspeckle assembly transcript 1; EMT, epithelial to mesenchymal transition. 
Recently, the competing endogenous RNA (ceRNA) hypothesis has suggested that IncRNA might function as a molecular sponge of miRNAs to regulate target gene expression, thereby playing an important role in the tumorigenic process. ${ }^{26}$ Hence, online software (Lncbase v.2) was used to predict target miRNAs that interact with NEAT1. Among these candidates, miR-34a-5p was chosen for further research. We demonstrated that NEAT1 represses miR-34a-5p expression by directly binding to miR-34a-5p and that NEAT1 knockdown exerts suppression effects on cell proliferation, migration, invasion, and EMT via miR-34a-5p. All these data hinted that NEAT1 might act as a ceRNA of miR-34a-5p.

MiR-34a-5p has been reported to act as a tumor suppressor in several human malignant tumors. For instance, miR-34a$5 p$ was downregulated in colorectal cancer (CRC) tissues, and miR-34a-5p repressed CRC recurrence and metastasis depending on p53 levels. ${ }^{27}$ Upregulated miR-34a-5p was shown to lead to repression of cell viability, migration, and invasion and to promote cell apoptosis and inactivation of Wnt/ $\beta$-catenin signaling pathway by targeting Sirtl in osteosarcoma. ${ }^{28}$ Additionally, miR-34a was reported to abrogate TGF- $\beta$ induced EMT by targeting SMAD4 in NPC cells. ${ }^{29}$ Moreover, researchers indicated that miR-34a-5p expression was reduced by the IncRNA XIST, which exerts oncogenic functions in NPC, and XIST-mediated oncogenic function was abated partially by miR-34a-5p, indicating that miR-34a-5p might act as a tumor suppressor of NPC..$^{30}$ Conversely, Maroni, et al. ${ }^{31}$ reported that miR-34a-5p was upregulated in non-metastatic ductal breast carcinoma and was implicated in the bone-metastatic process. Huang, et al. ${ }^{29}$ also reported that miR-34a expression was elevated during DNA damage response in chronic lymphocytic leukemia (CLL) and that low miR-34a levels were positively correlated with worse prognosis in CLL patients.

Abnormal activation of Wnt/ $\beta$-catenin signaling pathway has been shown to trigger tumorigenesis and progression in a large number of human cancers. ${ }^{19} \mathrm{Wnt} / \beta$-catenin signaling was described as being strongly correlated with O6-methylguanine-DNA methyltransferase (MGMT) expression; moreover, its suppression was found to enhance the effects of alkylating drugs and restore chemosensitivity in multiple cancers. ${ }^{32}$ Researchers also reported that activation of Wnt/ $\beta$-catenin signaling pathway promotes the proliferation of gastric cancer stem cells (CSCs). ${ }^{33}$ Blockade of Wnt $/ \beta$-catenin signaling was shown to inhibit metastasis and systemic tumor dissemination in breast cancer, providing a promising therapeutic target for breast cancer. ${ }^{34}$ In the present study, we found that NEATl knockdown blockaded Wnt/ $\beta$-catenin pathway by miR-34a$5 p$ in NPC cells and that NEAT1 knockdown repressed tumor growth and EMT by blockading Wnt/ $\beta$-catenin pathway in vivo. Similar with our findings, Zhang, et al.$^{35}$ reported that Yippee-like 3 hindered the metastasis and EMT of NPC cells by inhibiting Wnt/ $\beta$-catenin signaling pathway. Wang, et al. ${ }^{36}$ verified that ZNRF3 repressed the tumorigenesis and invasion in NPC through blockading of the Wnt/ $\beta$-catenin signaling pathway.

In conclusion, our study demonstrated that NEAT1 is elevated in NPC tissues and cell lines. Furthermore, we discovered that NEAT1 targets miR-34a-5p, at least in part, to drive NPC progression by regulating Wnt/ $\beta$-catenin signaling pathway, providing a new potential therapeutic target for NPC treatment.

\section{AUTHOR CONTRIBUTIONS}

Conceptualization: Yuqing Ji. Data curation: Man Wang. Formal analysis: Yuqing Ji. Funding acquisition: Man Wang. Investigation: Yuqing Ji, Man Wang. Methodology: Xueshen Li. Software: Xueshen Li. Supervision: Yuqing Ji. Writing - original draft: Fusheng Cui. Writing_-review \& editing: Fusheng Cui.

\section{ORCID iDs}

Yuqing Ji

Man Wang

Xueshen Li

Fusheng Cui https://orcid.org/0000-0003-1170-1278

https://orcid.org/0000-0003-4692-4578

https://orcid.org/0000-0001-9478-6777

https://orcid.org/0000-0002-8272-8649

\section{REFERENCES}

1. Lee VH, Lam KO, Chang AT, Lam TC, Chiang CL, So TH, et al. Management of nasopharyngeal carcinoma: is adjuvant therapy needed? J Oncol Pract 2018;14:594-602.

2. Yip TT, Ngan RK, Fong AH, Law SC. Application of circulating plasma/serum EBV DNA in the clinical management of nasopharyngeal carcinoma. Oral Oncol 2014;50:527-38.

3. Wu F, Wang R, Lu H, Wei B, Feng G, Li G, et al. Concurrent chemoradiotherapy in locoregionally advanced nasopharyngeal carcinoma: treatment outcomes of a prospective, multicentric clinical study. Radiother Oncol 2014;112:106-11.

4. Lai SZ, Li WF, Chen L, Luo W, Chen YY, Liu LZ, et al. How does intensity-modulated radiotherapy versus conventional two-dimensional radiotherapy influence the treatment results in nasopharyngeal carcinoma patients? Int J Radiat Oncol Biol Phys 2011; 80:661-8.

5. Spizzo R, Almeida MI, Colombatti A, Calin GA. Long non-coding RNAs and cancer: a new frontier of translational research? Oncogene 2012;31:4577-87.

6. Schmitt AM, Chang HY. Long noncoding RNAs in cancer pathways. Cancer Cell 2016;29:452-63.

7. Bhan A, Mandal SS. LncRNA HOTAIR: a master regulator of chromatin dynamics and cancer. Biochim Biophys Acta 2015;1856: 151-64.

8. Schmitt AM, Chang HY. Gene regulation: long RNAs wire up cancer growth. Nature 2013;500:536-7.

9. Choudhry H, Albukhari A, Morotti M, Haider S, Moralli D, Smythies J, et al. Tumor hypoxia induces nuclear paraspeckle formation through HIF-2 $\alpha$ dependent transcriptional activation of NEAT1 leading to cancer cell survival. Oncogene 2015;34:4482-90.

10. Wang P, Wu T, Zhou H, Jin Q, He G, Yu H, et al. Long noncoding RNA NEAT1 promotes laryngeal squamous cell cancer through regulating miR-107/CDK6 pathway. J Exp Clin Cancer Res 2016; 
35:22.

11. Fu JW, Kong Y, Sun X. Long noncoding RNA NEAT1 is an unfavorable prognostic factor and regulates migration and invasion in gastric cancer. J Cancer Res Clin Oncol 2016;142:1571-9.

12. Chai Y, Liu J, Zhang Z, Liu L. HuR-regulated lncRNA NEAT1 stability in tumorigenesis and progression of ovarian cancer. Cancer Med 2016;5:1588-98.

13. Cheng N, Guo Y. Long noncoding RNA NEAT1 promotes nasopharyngeal carcinoma progression through regulation of miR-

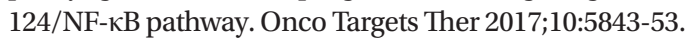

14. Lu Y, Li T, Wei G, Liu L, Chen Q, Xu L, et al. The long non-coding RNA NEAT1 regulates epithelial to mesenchymal transition and radioresistance in through miR-204/ZEB1 axis in nasopharyngeal carcinoma. Tumour Biol 2016;37:11733-41.

15. Chen Y, Gao DY, Huang L. In vivo delivery of miRNAs for cancer therapy: challenges and strategies. Adv Drug Deliv Rev 2015;81: 128-41.

16. Cheng JZ, Chen JJ, Wang ZG, Yu D. MicroRNA-185 inhibits cell proliferation while promoting apoptosis and autophagy through negative regulation of TGF- $\beta 1 / \mathrm{mTOR}$ axis and HOXC6 in nasopharyngeal carcinoma. Cancer Biomark 2018;23:107-23.

17. Zhu X, Li W, Zhang R, Liu Y. MicroRNA-342 inhibits cell proliferation and invasion in nasopharyngeal carcinoma by directly targeting ZEB1. Oncol Lett 2018;16:1298-304.

18. Zhang P, Hong H, Sun X, Jiang H, Ma S, Zhao S, et al. MicroRNA$10 \mathrm{~b}$ regulates epithelial-mesenchymal transition by modulating KLF4/Notch1/E-cadherin in cisplatin-resistant nasopharyngeal carcinoma cells. Am J Cancer Res 2016;6:141-56.

19. Fodde R, Brabletz T. Wnt/beta-catenin signaling in cancer stemness and malignant behavior. Curr Opin Cell Biol 2007;19:150-8.

20. Fu M, Zou C, Pan L, Liang W, Qian H, Xu W, et al. Long noncoding RNAs in digestive system cancers: functional roles, molecular mechanisms, and clinical implications (review). Oncol Rep 2016; 36:1207-18.

21. Jin C, Yan B, Lu Q, Lin Y, Ma L. The role of MALAT1/miR-1/slug axis on radioresistance in nasopharyngeal carcinoma. Tumour Biol 2016;37:4025-33.

22. Ma DD, Yuan LL, Lin LQ. LncRNA HOTAIR contributes to the tumorigenesis of nasopharyngeal carcinoma via up-regulating FASN. Eur Rev Med Pharmacol Sci 2017;21:5143-52.

23. Yang L, Tang Y, He Y, Wang Y, Lian Y, Xiong F, et al. High expression of LINC01420 indicates an unfavorable prognosis and modulates cell migration and invasion in nasopharyngeal carcinoma. J Cancer 2017;8:97-103.
24. Sun Q, Liu H, Li L, Zhang S, Liu K, Liu Y, et al. Long noncoding RNA-LET, which is repressed by EZH2, inhibits cell proliferation and induces apoptosis of nasopharyngeal carcinoma cell. Med Oncol 2015;32:226.

25. Chak WP, Lung RW, Tong JH, Chan SY, Lun SW, Tsao SW, et al. Downregulation of long non-coding RNA MEG3 in nasopharyngeal carcinoma. Mol Carcinog 2017;56:1041-54.

26. Liz J, Esteller M. IncRNAs and microRNAs with a role in cancer development. Biochim Biophys Acta 2016;1859:169-76.

27. Gao J, Li N, Dong Y, Li S, Xu L, Li X, et al. miR-34a-5p suppresses colorectal cancer metastasis and predicts recurrence in patients with stage II/III colorectal cancer. Oncogene 2015;34:4142-52.

28. Jia D, Niu Y, Li D, Liu Z. IncRNA C2datl promotes cell proliferation, migration, and invasion by targeting miR-34a-5p in osteosarcoma cells. Oncol Res 2018;26:753-64.

29. Huang G, Du MY, Zhu H, Zhang N, Lu ZW, Qian LX, et al. MiRNA34a reversed TGF- $\beta$-induced epithelial-mesenchymal transition via suppression of SMAD4 in NPC cells. Biomed Pharmacother 2018;106:217-24.

30. Song P, Ye LF, Zhang C, Peng T, Zhou XH. Long non-coding RNA XIST exerts oncogenic functions in human nasopharyngeal carcinoma by targeting miR-34a-5p. Gene 2016;592:8-14.

31. Maroni P, Puglisi R, Mattia G, Carè A, Matteucci E, Bendinelli P, et al. In bone metastasis miR-34a-5p absence inversely correlates with Met expression, while Met oncogene is unaffected by miR34a-5p in non-metastatic and metastatic breast carcinomas. Carcinogenesis 2017;38:492-503.

32. Wickström M, Dyberg C, Milosevic J, Einvik C, Calero R, Sveinbjörnsson $B$, et al. Wnt/ $\beta$-catenin pathway regulates MGMT gene expression in cancer and inhibition of Wnt signalling prevents chemoresistance. Nat Commun 2015;6:8904.

33. Mao J, Fan S, Ma W, Fan P, Wang B, Zhang J, et al. Roles of Wnt/ $\beta$-catenin signaling in the gastric cancer stem cells proliferation and salinomycin treatment. Cell Death Dis 2014;5:e1039.

34. Jang GB, Kim JY, Cho SD, Park KS, Jung JY, Lee HY, et al. Blockade of Wnt/ $\beta$-catenin signaling suppresses breast cancer metastasis by inhibiting CSC-like phenotype. Sci Rep 2015;5:12465.

35. Zhang J, Wen X, Ren XY, Li YQ, Tang XR, Wang YQ, et al. YPEL3 suppresses epithelial-mesenchymal transition and metastasis of nasopharyngeal carcinoma cells through the Wnt/ $\beta$-catenin signaling pathway. J Exp Clin Cancer Res 2016;35:109.

36. Wang Z, Wang Y, Ren H, Jin Y, Guo Y. ZNRF3 inhibits the invasion and tumorigenesis in nasopharyngeal carcinoma cells by inactivating the Wnt/ $\beta$-catenin pathway. Oncol Res 2017;25:571-7. 